\title{
N91-28105
}

\section{AEROSOL SPECKLE EFFECTS ON ATMOSPHERIC PULSED LIDAR BACKSCATTERED SIGNALS}

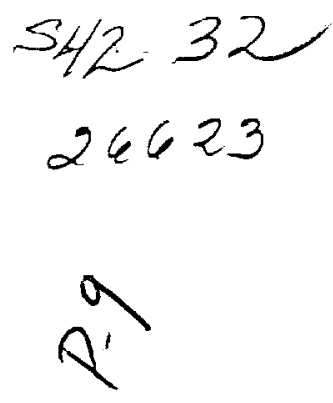

\author{
S.R. Murty \\ Alabama A\&M University \\ School of Engineering and Technology \\ Normal, Alabama
}

The effects of refractive turbulence along the path on the aerosol speckle field propagation and on the decorrelation time are studied for coherent pulsed lidar systems.

\section{Introduction}

Lidar systems using atmospheric aerosols as targets exhibit return signal amplitude and power fluctuations which indicate speckle effects. ${ }^{1,2}$ The speckle effects are manifested statistically as Rayleigh-distributed amplitude and exponentially distributed power of the backscattered signals. The signals from the various scatterers which make up the target combine to form a speckle pattern at any point along the backscattered beam. The speckle size is $\approx \lambda z / b$, where $\mathrm{z}$ is the range from the target, $\lambda$ is the wavelength, and $b$ is the radius of the illuminating beam on the diffuse target. Therefore, as the beam size on the diffuse target increases, the speckle size at the receiver decreases. Since the field across a speckle pattern is produced by the addition of a number of signals of random phase, the speckle pattern changes as each particle moves, thereby generating spatial and temporal changes in intensity. The particles move primarily due to atmospheric turbulence, and the changes in the speckle pattern are caused by the velocity turbulence in the illuminated region of the atmosphere which contributes to the backscattered signal at the receiver. For a pulsed laser, the backscatter region of the atmosphere is $c t_{p} / 2$ around the range point where $t_{p}$ is the pulse duration and $\mathrm{c}$ is the speed of light.

In addition to producing signal intensity fluctuations, aerosol-generated speckle causes a decorrelation of the backscattered signals, as the aerosols are free to move about relative to each other along the beam axis and transverse to it. The resulting decorrelation time determines the temporal window of the return signal from which the power spectrum can be calculated to obtain wind speed.

A laser beam experiences fluctuations in intensity and a reduction in coherence due to propagation in the refractive turbulence of the atmosphere between the telescope range point as shown in Fig. 1. Consequently the received signal characteristics are affected by a combination of refractive turbulence and speckle effects. The objective of this paper is to examine the effects of refractive turbulence along the path on the aerosol speckle field propagation and of the decorrelation time. 
Churnside and Yura ${ }^{3}$ have developed the basic theoretical formulation of the spatiotemporal correlation function for the aerosol return-signal field. Ancellet and Menzies ${ }^{4}$ presented results of measurement using a pulsed TEA $\mathrm{CO}_{2}$ laser and found a decorrelation time of $2-2.5 \mu$ s over a $1.3-\mathrm{km}$ range. Laser pulses shorter than $\sim 150$ ps are broadened due to atmospheric turbulence, but the pulse spreading and wander in turbulence decrease as the pulse becomes longer. ${ }^{5,6}$ In this work, the pulse is represented as a Fourier integral, and we make use of the two-frequency mutual coherence function to study propagation of the aerosol speckle field.

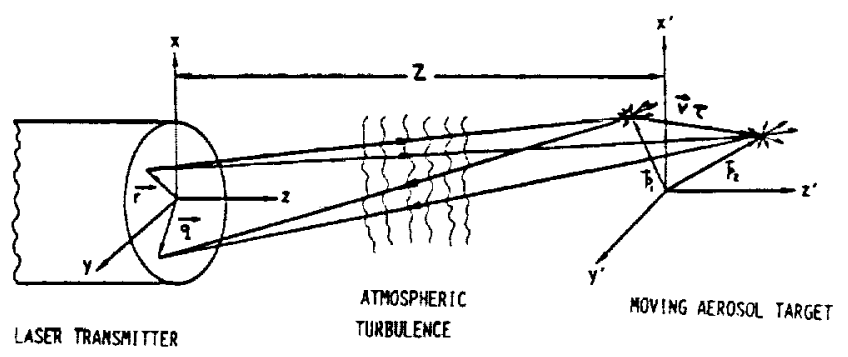

Fig. 1. Sketch of pulsed lidar propagating through atmospheric turbulence using moving aerosols as a target.

\section{Analysis}

The transmitter and receiver are assumed to be colocated having a common telescope aperture diameter $\mathrm{d}$. The laser transmitter is assumed to be pulsed with a pulse duration $t_{p}$ and a transmitted power $P_{T}(t)$ at time $t$. The complex field of the pulse propagating in the turbulent medium at any range point $\mathrm{z}$ is given by

$$
U_{I}(z, \mathbf{p}, t)=\int_{-\infty}^{\infty} A(\omega) u_{I}(\mathbf{p}, k, t) \exp (i \omega t) d \omega,
$$

where $A(\omega)$ is the Fourier spectral amplitude, $u_{I}$ is the monochromatic field amplitude at a distance $\mathrm{z}$ from the transmitter, $p$ is a 2-D transverse vector in the aerosol target plane, and $k$ is the optical wave number. Using the paraxial approximation and the extended Huygens-Fresnel principle, ${ }^{7} u_{I}$ can be expressed as

$$
\begin{aligned}
u_{I}(\mathbf{p}, k, t)=\frac{k \exp (i k z)}{2 \pi i z} \int d^{2} \mathbf{r} u_{T}\left(\mathbf{r}, t-\frac{z}{c}\right) \\
\quad \times \exp \left[\frac{i k}{2 z}(\mathbf{p}-\mathbf{r})^{2}+\psi(\mathbf{r}, \mathbf{p}, k, t)\right] .
\end{aligned}
$$

In Eq. (2), $\mathbf{r}$ is a transverse vector in the transmitter plane, $\psi(\mathbf{r}, \mathbf{p}, k, t)$ is the sum of the logamplitude and phase perturbation experienced by a spherical wave propagating from $\mathbf{r}$ to $\mathbf{p}$ through the atmospheric turbulence, and $u_{T}$ represents the field amplitude at the transmitter given by

$$
U_{T}(r, t)=\left[\frac{2 P_{T}(t)}{\pi a^{2}}\right]^{1 / 2} \exp \left[-\left(\frac{1}{a^{2}}+\frac{i k}{2}\right) r^{2}\right]
$$

where $\mathrm{a}$ is the beam radius at $\exp (-2)$ intensity, and $\mathrm{f}$ is the geometric focus of the telescope. 
The complex field $U_{I}(z, \mathbf{p}, t)$ is assumed to be backscattered at range $\mathrm{z}$ by an atmospheric aerosol present in the illuminated volume. The scattered pulse propagates back to the receiver through atmospheric turbulence, and its field is given by

$$
U_{s}(\mathbf{q}, t)=\int_{-\infty}^{\infty} A(\omega) u_{s}(\mathbf{q}, k, t) \exp (i \omega t) d \omega
$$

where the monochromatic wave field $u_{s}(q, k, t)$ is given by ${ }^{9}$

$$
\begin{aligned}
u_{s}(\mathbf{q}, k, t)= & \left.\frac{S(k)}{z} u_{I}\left(\mathbf{p}, k, t-\frac{z}{c}\right)\right) \\
& \times \exp \left[i k z+\frac{i k}{2 z}(\mathbf{q}-\mathbf{p})^{2}+\psi(\mathbf{p}, \mathbf{q}, k, t)\right] .
\end{aligned}
$$

In Eq. (5), $q$ is a transverse vector in the receiver plane and $S(k)$ is the monochromatic backscatter coefficient of the aerosol.

The pulse is assumed to have a center carrier angular frequency $\omega_{0}$ and optical wavenumber $k_{0}$ and is narrowband. It is convenient to write Eq. (4) as

$$
\begin{aligned}
U_{s}(\mathbf{q}, t)= & \exp \left[i\left(\omega_{0} t-k_{0} z\right)\right] \int_{-\infty}^{\infty} d \omega A\left(\omega+\omega_{0}\right) \\
& \times u_{s}\left(q, \omega+\omega_{0}, t\right) \exp (i \omega t) .
\end{aligned}
$$

During a small time interval of $<1 \mathrm{~ms}$, the aerosol position is assumed to change due to a radial velocity $v_{z}$, a transverse velocity $\mathbf{V}_{T}$ to a new position given by the following relations:

$$
\begin{aligned}
& z_{\tau}=z(t+\tau)+z(t)+v_{z} \tau \\
& \mathbf{p}_{\boldsymbol{\tau}}=\mathbf{p}(t+\tau)=\mathbf{p}(t)+\mathbf{V}_{T} \tau
\end{aligned}
$$

The backscattered field at time $t+\tau$, is given by

$$
\begin{aligned}
U_{s}(\mathbf{q}, t+\tau)= & \exp \left[i \omega_{0}\left(t+\tau-z_{\tau}\right)\right] \int_{-\infty}^{\infty} A\left(\omega+\omega_{0}\right) \\
& \times u_{s}\left(\mathbf{q}, \omega+\omega_{0}, t+\tau\right) \\
& \times \exp \left[i \omega\left(t+\tau-z_{\tau / c}\right) d \omega,\right.
\end{aligned}
$$

and the monochromatic wave field at time and $t+\tau$ is given by 


$$
\begin{aligned}
u_{s}(\mathbf{q}, \omega, t+\tau)= & \frac{k S \exp \left(2 i k z_{\tau}\right)}{2 \pi i z^{2}} \int d r^{2} u_{T}\left(\mathbf{r}, t+\tau-\frac{2 z}{c}\right) \\
& \times \exp \left[\frac{i k}{2 z}\left(\mathbf{p}_{\tau}-\mathbf{r}\right)^{2}+\left(\mathbf{q}-\mathbf{p}_{\tau}\right)^{2}\right] \\
& +\Psi\left(\mathbf{r}, \mathbf{p}_{\tau}, k, t+6 \tau\right)+\Psi\left(\mathbf{p}_{\tau}, \mathbf{q}, k, t+\tau\right)
\end{aligned}
$$

The cross-product of the received fields at time $t$ and $t+\tau$ is given by

$$
\begin{aligned}
U_{s}\left(\mathbf{q}_{1}, t\right) U_{s}^{*}\left(\mathbf{q}_{2}, t+\tau\right)= & \int_{-\infty}^{\infty} d \omega_{1} \int_{-\infty}^{\infty} d \omega_{2} A_{e}\left(\omega_{1}\right) \\
& \times u_{s}\left(\mathbf{q}_{1}, \omega_{1}, t\right) A_{e}\left(\omega_{2}\right) u_{s}^{*}\left(\mathbf{q}_{2}, \omega_{2}, t+\tau\right) \\
& \times \exp \left[i\left(\omega_{1}-\omega_{2}\right) t\right]
\end{aligned}
$$

where $A_{e}(\omega)=A\left(\omega+\omega_{0}\right)$.

Equation (9) will be ensemble-averaged over the propagation path for refractive turbulence and over the illuminated volume for velocity turbulence to obtain the pulse spatiotemporal correlation function. This process is represented by double angular brackets, and the correlation function of the received optical fields is given by

$$
\begin{aligned}
\ll U_{s}\left(\mathbf{q}_{1}, t\right) U_{s}^{*}\left(\mathbf{q}_{2}, t+\tau\right) \gg= & \int_{-\infty}^{\infty} d \omega_{1} \int_{-\infty}^{\infty} d \omega_{2} A_{e}\left(\omega_{1}\right) A_{e}^{*}\left(\omega_{2}\right) \\
& \times \Gamma\left(\mathbf{q}_{1}, \mathbf{q}_{2}, \omega_{1}, \omega_{2}\right) \\
& \times \exp \left[i\left(\omega_{1}-\omega_{2}\right) t\right]
\end{aligned}
$$

where $\Gamma\left(\mathbf{q}_{1}, \mathbf{q}_{2}, \omega_{1}, \omega_{2}\right)=\ll u_{s}\left(\mathbf{q}_{1}, \omega_{1}, t\right) u_{s}\left(\mathbf{q}_{2}, \omega_{2}, t+\tau\right) \gg$ is a two-frequency mutual coherence function. The determination of the effects of aerosol speckle, refractive turbulence, and pulse shape are now dependent on the evaluation of the function $\Gamma$.

\section{Ensemble Averaging}

Following Churnside and Yura ${ }^{3}$ for the averaging over velocity turbulence, the aerosol velocities are assumed Gaussian-distributed within the inertial subrange, and we obtain the expected value of the mutual coherence function as

$$
\begin{aligned}
\Gamma\left(\mathbf{q}_{1}, \mathbf{q}_{2}, \omega_{1}, \omega_{2}\right)= & \int_{-\infty}^{\infty} d v_{z} \int_{-\infty}^{\infty} d^{2} \mathbf{v}_{T} \int d z d^{2} \mathbf{p} \\
& \times u_{s}\left(\mathbf{q}_{1}, \omega_{1}, t\right) u_{s}^{*}\left(\mathbf{q}_{2}, \omega_{2} t+\tau\right) \\
& \times P\left(v_{z}\right) P\left(\mathbf{V}_{T}\right) / V
\end{aligned}
$$


In Eq. (11), V is the illuminated volume, and the probability density functions of the velocity components are given by

$$
\begin{gathered}
P\left(v_{z}\right)=\frac{1}{\sqrt{2 \pi \sigma_{z}}} \exp \left[-\left(v_{z}-\bar{\nabla}_{z}\right)^{2} / 2 \sigma_{z}^{2}\right], \\
P\left(\mathbf{V}_{T}\right)=\frac{1}{2 \pi \sigma_{T}^{2}} \exp \left[-\left(\mathbf{V}_{T}-\nabla_{T}\right)^{2} \sigma_{T}^{2}\right],
\end{gathered}
$$

where $\bar{\nu}_{z}, \nabla_{T}$ and $\sigma_{z}^{2}, \sigma_{T}^{2}$ are the means and variances of the wind components along the $\mathrm{z}$ axis and transverse to it.

The ensemble average over path turbulence is given by

$$
\begin{aligned}
\left\langle u_{s}\left(\mathbf{q}_{1}, \omega_{1}, t\right) u_{s}^{*}\left(\mathbf{q}_{2}, \omega_{2}, t+\tau\right)\right\rangle= & \frac{k_{1} k_{2} S\left(\omega_{1}\right) S\left(\omega_{2}\right)}{\left(2 \pi z^{2}\right)^{2}} \exp \left[2 i\left(k_{1}-k_{2}\right) z\right. \\
& \left.-2 i k_{2} v_{z} \tau\right] \iint d^{2} \mathbf{r}_{1} d^{2} \mathbf{r}_{2} u_{T}\left(\mathbf{r}_{1}, t-\frac{2 z}{c}\right) \\
& \times u_{T}^{*}\left(\mathbf{r}_{2}, t+\tau-\frac{2 z}{c}\right) \exp \left[\frac{i k_{1}}{2 z}\left(\mathbf{p}-\mathbf{r}_{1}\right)^{2}\right. \\
& +\frac{i k_{1}}{2 z}\left(\mathbf{q}_{1}-\mathbf{p}\right)^{2}-\frac{i k_{2}}{2 z}\left(\mathbf{p}+\mathbf{V}_{T} \tau-\mathbf{r}_{2}\right)^{2} \\
& \left.-\frac{i k_{2}}{2 z}\left(q_{2}-\mathbf{p}-V_{T} \tau\right)^{2}\right] \\
& \times H\left(\mathbf{q}_{1}, \mathbf{q}_{2}, \mathbf{r}_{1}, \mathbf{r}_{2}, k_{1}, k_{2}\right),
\end{aligned}
$$

where $\mathrm{H}$ is the fourth-order mutual coherence function given by

$$
\begin{aligned}
H= & \left(\operatorname { e x p } \left[\Psi\left(\mathbf{p}, \mathbf{r}_{1}, k_{1}, t\right)+\Psi\left(\mathbf{p}, \mathbf{q}_{1}, k_{1}, t\right)+\Psi^{*}\left(\mathbf{p}+\mathbf{V}_{T} \tau, \mathbf{r}_{2}, k_{2}, t+\tau\right)\right.\right. \\
& \left.+\Psi^{*}\left(\mathbf{p}+\mathbf{V}_{T} \tau, \mathbf{q}_{2}, k_{2}, t+\tau\right)\right\rangle
\end{aligned}
$$

The change in the aerosol position has very little effect on the refractive turbulence encountered along the path for small $\tau$ and will be neglected in evaluating the mutual coherence function, which can be written as ${ }^{8}$

$$
\begin{aligned}
H= & \exp \left[-\left(V_{2}\right)\left(D_{12}-D_{13}+D_{14}+D_{23}+D_{34}-D_{24}+D_{34}\right)\right. \\
& \left.+2 C_{X_{12}}+2 C_{X_{34}}\right] .
\end{aligned}
$$

In Eq. (14) $D_{i j}=D\left(\left|\mathbf{r}_{i}-\mathbf{r}_{j}\right|\right)$ is the two-frequency wave structure function of a point source given by ${ }^{10}$ 


$$
\begin{aligned}
D(\rho) & =2\left(\rho / \rho_{0}\right)^{5 / 3}-2 \Delta_{12}^{2} / \Omega^{2}, \\
\rho_{0} & =\left(0.545 k_{0} C_{n}^{2} z\right)^{-3 / 3}, \\
\Omega^{-2} \simeq 0.39 k_{0} C_{n}^{2} L_{o}^{5 / 3} z, \Delta_{12} & =\left|k_{1}-k_{2}\right| / k_{0},
\end{aligned}
$$

where $L_{0}$ is the outer scale of turbulence, $\mathrm{c}$ is the speed of light, and $C_{n}^{2}$ is the turbulence structure constant assumed uniform along the path. $C_{x i j}=C_{x}\left(\left|\mathbf{r}_{i}-\mathbf{r}_{j}\right|\right)$ is the two-frequency covariance of the logamplitude of a point source given by ${ }^{11}$

$$
\begin{aligned}
C_{x i j}= & 0.132 \pi^{2} k_{1} k_{j} z C_{n}^{2} \int_{0}^{1} d t \int u u^{-8 / 3} J_{0} \\
& \times\left[u\left|\left(\mathbf{r}_{i}-\mathbf{r}_{j}\right)(1-t)\right|\right] \sin \left[\frac{u^{2} t(1-t) z}{2 k_{1}}\right] \\
& \times \sin \left[\frac{u^{2} t(1-t) z}{2 k_{j}}\right] .
\end{aligned}
$$

The integrations of Eq. (12) are lengthy and cannot be performed in closed form. We present the approximate results in this work for the simplified case of a collimated beam and negligible transverse velocity component and consider points along the beam axis. It is also assumed that $\Delta_{12} \ll 1$, and we make use of a quadratic approximation for the structure function. With these simplications, the integrations of Eq. (12) can be carried out, and the result is

$$
\begin{aligned}
\left\langle u_{s}\left(o, \omega_{1}, t\right) u_{s}^{*}\left(o, \omega_{2}, t+\tau\right)\right\rangle= & \frac{2 S^{2} F^{2}}{a^{2} z^{2}\left(1+F^{2}+2 a^{2} / \rho_{0}^{2}\right) \pi} \\
& \times\left[P_{T}\left(t-\frac{62 z}{c}\right) P_{T}\left(t+\tau-\frac{2 z}{c}\right)\right]^{v_{2}} \\
& \times \exp \left\{i\left(\omega_{1}-\omega_{2}\right) T_{d}-\left(\omega_{1}-\omega_{2}\right)^{2 / \Omega^{2}}\right. \\
& -\left(k_{1}-k_{2}\right) p^{2} / 2 z-2\left(\frac{k_{0} a^{2}}{2 z}\right) p^{2} / \\
& {\left.\left[1+\left(\frac{k_{0} a^{2}}{2 z}\right)\right]^{2} 2 i k_{1} z-2 i k_{2} z \tau\right\} }
\end{aligned}
$$

where $F=k_{0} a^{2} / 2 z$ is the Fresnel number of the source, $T_{d}$ si the time delay given by

$$
T_{d} \approx \frac{F}{c k_{0}}\left(\frac{1+a^{2} / \rho_{0}^{2}}{1+F^{2}+2 a^{2} / \rho_{0}^{2}}\right)
$$


the coherence bandwidth is given by

$$
\Omega^{-2} \approx 0.39 C_{n}^{2} L_{o}^{5 / 3} z / c^{2}
$$

and $L_{o}$ is the outer scale of the turbulence, and $\mathrm{c}$ is the speed of light. It may be noted that the pulse broadening is inversely proportional to the coherence bandwidth. We will now carry out integrations indicated in Eq. (11) over the velocity spectrum and sensing volume $\mathrm{V}$ and replace $S^{2} N / V$ by the aerosol backscatter coefficient $\beta$, where $\mathrm{N}$ is the total number of aerosols within the volume $\mathrm{V}$. The result is

$$
\begin{aligned}
\Gamma\left(o, o, \omega_{1}, \omega_{2}\right)= & \int \frac{\beta}{2 z^{2}}\left[P_{T}\left(t \frac{2 z}{c}\right) P_{T}\left(t+\tau-\frac{2 z \tau}{c}\right)\right] v_{2} \\
& \times \exp \left[i\left(\omega_{1}-\omega_{2}\right) T_{d}-\left(\omega_{1}-\omega_{2}\right)^{2} / \Omega^{2}\right. \\
& \left.+2 i\left(k_{1}-k_{2}\right) z-2 i k_{2} \bar{v}_{z} \sigma_{z} \tau-2 \sigma_{z}^{2} k_{2}^{2} \tau^{2}\right] d z
\end{aligned}
$$

The integrations over $\omega_{1}$ and $\omega_{2}$ are carried out for a Gaussian pulse with frequency spectrum given by

$$
A(\omega)=\left(2 \pi \sigma_{\omega}^{2}\right)^{-1 / 2} \exp \left[\left(\omega-\omega_{0}\right)^{2} / 2 \sigma_{\omega}^{2}\right]
$$

where $\sigma_{\omega}$ is the bandwidth of the pulse. The variables are changed using $2 \omega_{0}^{\prime}=\omega_{1}+\omega_{2}$, $\delta=\omega_{1}-\omega_{2}$.

The spatiotemporal correlation function is calculated for a Gaussian transmitted power distribution given by

$$
P_{T}(t)=\frac{E}{\sqrt{\pi} t_{p}} \exp \left(-t^{2} / t_{p}^{2}\right)
$$

where $E$ is the total energy in the pulse, and the pulse width is related to bandwidth as $t_{p}=\sqrt{2} \sigma_{\omega}^{-1}$. The integration over $\mathrm{z}$ is performed designating arbitrarily the time of peak power by $\mathrm{t}=$ 0 , and the result is 


$$
\begin{aligned}
\ll U_{s}(o, t) U_{s}^{*}(o, t+\tau) \gg= & \frac{c \beta E}{2 z_{0}^{2}} \frac{\Omega}{\left(\Omega^{2}+4 \sigma_{\omega}^{2}\right)^{v_{2}}} \frac{\tau_{e}}{\left(\tau_{e}^{2}+t_{p}^{2}\right)^{v_{2}}} \\
& \times \frac{1}{1+6 F^{2}+2 a^{2} / \rho_{0}^{2}} \exp \left[-2 \sigma_{z}^{2} k_{0}^{2} \tau^{2}\right. \\
& -\frac{\sigma_{\omega}^{2}}{4}\left(\tau^{2}+2 \bar{v}_{z} \sigma_{z} \tau / c\right)^{2}-\frac{\tau_{e}^{2}}{\tau_{e}^{2}+t_{p}^{2}} \\
& \times\left[\frac{\tau^{2}}{4 t_{p}^{2}}+\frac{\tau^{2} / 4+\left(T_{d}+\bar{v}_{z} \tau / c\right)^{2}}{\tau_{e}^{2}}\right] \\
& \left.-i\left(\omega_{0} \tau+2 k_{0} \bar{v}_{z} \tau\right)\right],
\end{aligned}
$$

where $\tau_{e}^{2}=\sigma_{\omega}^{-2}+4 \Omega^{-2}$ and $z_{0}$ is the range. Equation (22) is the main result of this paper, which exhibits the effects of refractive path turbulence on pulse spreading and aerosol speckle. The first term in the exponential gives the decorrelation effect due to velocity turbulence along the beam of the axis; the second and third terms give the effect of refractive turbulence and velocity turbulence on pulse broadening and its contribution to the decorrelation of aerosol scattered signals.

\section{Discussion}

The spatiotemporal correlation function obtained by Churnside and Yura ${ }^{3}$ has been extended to include the spectral effect of pulsed signals and refractive turbulence along the propagation path. An approximate result for a collimated beam along the beam axis is presented in Eq. (22) for the combined effects assuming a quadratic approximation for the structure functions. These approximations permit us to bring out explicitly the effect of time delay $T_{d}$ and coherence bandwidth $\Omega$ on the correlation function.

We consider a long path wind-measuring pulsed lidar having a pulse duration of $100 \mathrm{~ns}$ operating at $9.1 \mu \mathrm{m}$ wavelength. The path length is assumed as $100 \mathrm{~km}$ through a turbulent atmosphere with $C_{n}^{2}=6.4 \times 10^{-15} \mathrm{~m}^{-2 / 3}$ corresponding to a clear day in the lower atmosphere with an outer scale length $L_{0}=100 \mathrm{~m}$ and $\sigma_{z}=1 \mathrm{~m} / \mathrm{s}$. The beam radius at $\exp (-2)$ intensity is taken as $1 \mathrm{~m}$, which gives the Fresnel number of the source as $F=3.45$. The transverse coherence length for this path is $\rho_{0}=0.0465 \mathrm{~m}$.

The coherence bandwidth $\Omega$ and the time delay $T_{d}$ corresponding to these parameters are $\Omega=4.1 \times 10^{11} \mathrm{rad} / \mathrm{s}$ and $T_{d}=1.65 \times 10^{-14} \mathrm{~s}$. Using these values, we obtain $\sigma_{\omega}=1.4 \times 10^{10} \mathrm{rad} / \mathrm{s}$ and $\tau_{e}=70.9 \mathrm{~ns}$. These time scales are shorter than the correlation time of the order of $1 \mu \mathrm{s}$ corresponding to moderate turbulence-induced aerosol dephasing, and the effect of pulse broadening is negligible for this case. When the correlation time due to aerosol dephasing approaches the nanosecond range, pulse broadening effects need to be considered.

This work is supported by NASA grant NSG 8037. It is a pleasure to acknowledge the discussions with James W. Bilbro of NASA Marshall Space Flight Center. 


\section{References}

1. R.M. Hardesty, R.J. Keeler, M.J. Post, and R.A. Richter, "Characteristics of Coherent Lidar Returns from Calibration Targets and Aerosols," Appl. Opt. 20, 3763 (1981).

2. P.H. Flamant, R.T. Menzies, and M.J. Kavayaa, "Evidence for Speckle Effects on Pulsed $\mathrm{CO}_{2}$ Lidar Signal Returns form Remote Targets," Appl. Opt. 23, 1412 (1984).

3. H. Churnside and H.T. Yura, "Speckle Statistics of Atmospherically Backscattered Laser Lights," Appl. Opt. 22, 2559 (1983).

4. G.M. Ancellet and R.T. Menzies, "Atmospheric Correlation Time Measurements and Effects on Coherent Doppler Lidar," J. Opt. Soc. Am. A 4, 367 (1987).

5. C.H. Liu and K.C. Yeh, "Pulse Spreading and Wandering in Random Media," Rad. Sci, 14, 925, (1979).

6. I. Sreeivasiah, A. Ishimaru, and T.S. Hong, "Two Frequency Mutual Coherence Function and Pulse Propagation in a Random Medium: An Analytic Solution to the Plane Wave Case," Rad. Sci. 11, 775 (1976).

7. R.F. Lutomirski and H.T. Yura, "Propagation of a Finite Optical Beam in an Inhomogeneous Medium," Appl. Opt. 10, 1652 (1971).

8. H.M. Lee, J.F. Holmes, and J.R. Kerr, "Statistics of Speckle Propagation Through the Turbulent Atmosphere,” J. Opt. Soc. Am. 66, 1164 (1976).

9. H.T. Yura, "Mutual Coherence Function of a Finite Cross-Section Optical Beam Propagating in a Turbulent Medium," Appl. Opt. 11, 1399 (1972).

10. R.L. Fante, "Electromagnetic Beam Propagation in Turbulent Media: An Update," Proc. IEEE 68, 1424 (1980).

11. A. Ishimaru, "Temporal Frequency Spectra of Multifrequency Waves in Turbulent Atmosphere," IEEE Trans. Antennas Propag. AP-20, 10 (1972). 